Optical Bistability in Incident-Dependent Two-Dimensional Nonlinear Optical Superlattices

This article has been downloaded from IOPscience. Please scroll down to see the full text article. 1996 Chinese Phys. Lett. 13913

(http://iopscience.iop.org/0256-307X/13/12/010)

View the table of contents for this issue, or go to the journal homepage for more

Download details:

IP Address: 218.94.142.221

The article was downloaded on 16/02/2012 at 10:23

Please note that terms and conditions apply. 


\title{
Optical Bistability in Incident-Dependent Two-Dimensional Nonlinear Optical Superlattices *
}

\author{
CHEN Xiang-fei, LU Ya-lin, LU Yan-qing, MIN Nai-ben ${ }^{1}$ (N. B. Ming) \\ National Laboratory of Solid State Microstructures, Nanjing University, Nanjing 210093 \\ ${ }^{1}$ also CCAST (World Laboratory), P. O. Box 8730, Beijing 100080
}

\section{(Received 9 September 1996)}

\begin{abstract}
It is shown that, when two coherent waves are obliquely incident to a one-dimensional nonlinear optical superlattice containing Kerr-form dielectric nonlinearity, an incident-dependent twodimensional nonlinear optical superlattice can be constructed. This structure can be bistable. There exist two types of optical bistable mechanism, i. e., the index-modulation mechanism and distributed feedback mechanism. Owing to the index-modulation mechanism, the threshold for the bistability is lower than that of a more traditional one-dimensional distributed feedback structure.
\end{abstract}

\section{PACS: $42.65 . P c, 42.25 . F x$}

Since optical bistability in one-dimensional (1D) nonlinear superlattices was first discovered by Winful et al. more than ten years ago, ${ }^{1}$ the idea has been developed in a number of papers. ${ }^{2-7}$ Very recently, optical response in a two-dimensional (2D) nonlinear superlattice (a nonlinear dielectric medium with its refractive index periodically modulated in two dimensions) was also studied. Optical bistability and self-pulsing were discovered in 2D nonlinear superlattices, both theoretically and experimentally. ${ }^{8-9}$ In the presence of Kerr-form nonlinearity, the interference in the transmission field will perturb the index modulation (IM) strength. Thus a positive feedback is formed. This new type of bistable mechanism, named IM mechanism, has been demonstrated. ${ }^{8}$ In the present paper, with two coherent waves obliquely incident to a 1D nonlinear superlattice, we show that there exist two types of bistable mechanism, i.e., IM mechanism and distributed feedback (DFB) mechanism. Owing to the additional mechanism (IM mechanism), the bistable threshold can be lower than that of a DFB structure.

We consider an isotropic lossless nonlinear medium with its refractive index modulated periodically in one dimension. The periodicity is expressed by vector $H_{z}$ in reciprocal space. Then the one-dimensional linear and periodically modulated refractive index is defined by the following equation

$$
n=n_{0}+n_{z} \cos \left(H_{z} z\right),
$$

where $n_{z} \ll n_{0}$. Two coherent incident waves, with the same incident angle, are symmetrically incident to the 1D nonlinear superlattice, as shown in Fig. 1. In the presence of one incident wave, two diffracted waves will be excited in the 1D superlattice. Correspondingly, in the presence of two incident waves, four diffracted waves will be excited. The Kerr-form nonlinearity can be described by the nonlinear polarization term

$$
P_{\mathrm{NL}}(r)=\frac{1}{4 \pi} n_{0} n_{\alpha}|E|^{2} E
$$

where $n_{\alpha}$ is the nonlinear index. The following scalar Maxwell's equations describe the light transmission in the nonlinear medium:

$$
\left[\nabla^{2}+\frac{\omega^{2}}{c^{2}}\left(n^{2}+n_{0} n_{\alpha}\right)|E(\boldsymbol{r})|^{2}\right] E(\boldsymbol{r})=0,
$$

where $\omega$ is the light frequency, and $c$ the speed of light in vacuum. Near the Bragg condition, one can write the field in the medium as a sum of two forward and two backward diffracted waves

$E(z)=E_{0}(z) \exp \left(\mathrm{i} \boldsymbol{K}_{0} \cdot \boldsymbol{r}\right)+E_{\mathrm{h}}(z) \exp \left(\mathrm{i} \boldsymbol{K}_{\mathrm{h}} \cdot \boldsymbol{r}\right)+E_{-0}(z) \exp \left(-\mathrm{i} \boldsymbol{K}_{0} \cdot \boldsymbol{r}\right)+E_{-\mathrm{h}}(z) \exp \left(-\mathrm{i} \boldsymbol{K}_{\mathrm{h}} \cdot \boldsymbol{r}\right)$,

\footnotetext{
* Supported by a Grant for the Key Research Project in Climbing Program from the State Science and Technology Commission of China. (C) by the Chinese Physical Society
} 
where $K_{0}=K_{\mathrm{h}}=k_{\mathrm{m}}$, and $k_{\mathrm{m}}=n_{0} \omega / c$ is the wave number in the average refractive index. Furthermore, the Bragg condition leads to the following equation

$$
H_{z}=2 k_{\mathrm{m}} \cos \left(\theta_{\mathrm{B}}\right) \text {, }
$$

where $\theta_{\mathrm{B}}$ is the Bragg angle. The four diffracted waves are coherent, and thus obviously interfere each other in the nonlinear medium. This four-wave mixing process, through the Kerr-form nonlinearity, leads to the formation of the other periodical refractive index modulations along $x$, $x+\check{z}, x-z$ directions. Then a $2 \mathrm{D}$ periodically modulated structure is constructed. ${ }^{8}$ Compared with a pre-constructed $2 \mathrm{D}$ optical superlattice, the modulation parameters, i. e., modulation periodicities and modulation strengths, are mainly determined by the two incident waves. This incident-dependent (ID) periodically modulated structure is called the ID 2D nonlinear superlattice in the present paper. In the ID $2 \mathrm{D}$ nonlinear superlattice, the modulation strengths along $x, x+z, x-z$ directions are proportional to $\sqrt{I_{\text {in } 1} I_{\text {in } 2}}$. Where $I_{\text {in } 1}, I_{\text {in2 }}$ are intensities of the two incident waves. Therefore, there are two types of positive feedbacks in an ID 2D nonlinear superlattice. One is the well-known DFB, and the other is the IM feedback. Owing to the contribution of the refractive index modulation, the bistable threshold should be lower than that of a more traditional 1D DFB structure.

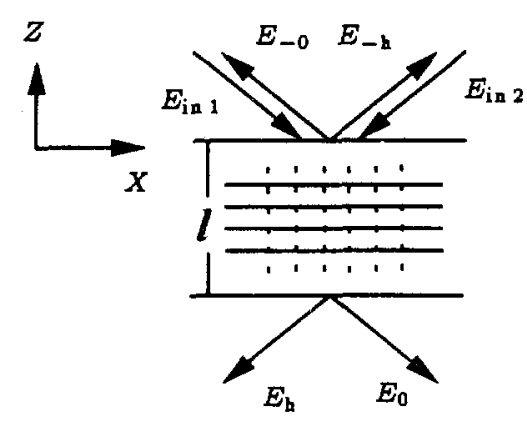

Fig. 1. Schematic diagram of four-wave diffraction in the ID $2 D$ nonlinear superlattice, the dotted lines represent the ID periodical modulation along $x$-direction.

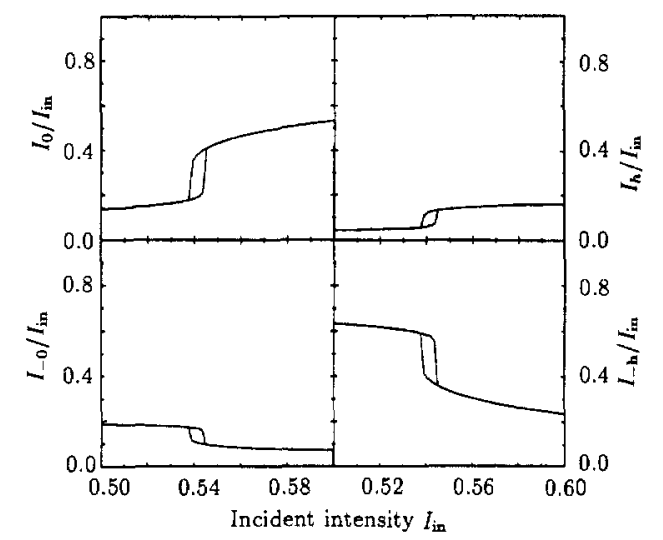

Fig. 2. Bistable behavior in the incidentdiffracted relations in ID $2 \mathrm{D}$ nonlinear superlattices obtained by step-input numerical solution. The structure parameters $\triangle \beta=0, M_{z}=$ $2 \times 10^{-4}, k_{\mathrm{m}} l / \cos \theta_{\mathrm{B}}=3.5 \times 10^{4}$, and $\gamma=0.3$, here $n_{\alpha} I_{\text {unit }}=10^{-4}$.

Inserting Eqs. (1), (2), (4), and (5) into Eq. (3), we obtain, in the slowly varying envelope approximation

$$
\begin{aligned}
\frac{\mathrm{d} E_{0}}{\mathrm{~d} z} & =-\mathrm{i} \frac{k_{\mathrm{m}}}{4 \cos \theta_{\mathrm{B}}}\left\{2 \delta n_{0} E_{0}+\delta n_{x} E_{\mathrm{h}}+\delta n_{x-z} E_{-0}+\left[M_{z} \exp (\mathrm{i} 2 \Delta \beta z)+\delta n_{z}^{*}\right] E_{-\mathrm{h}}\right\}, \\
\frac{\mathrm{d} E_{\mathrm{h}}}{\mathrm{d} z} & =-\mathrm{i} \frac{k_{\mathrm{m}}}{4 \cos \theta_{\mathrm{B}}}\left\{\delta n_{x}^{*} E_{0}+2 \delta n_{0} E_{\mathrm{h}}+\left[M_{z} \exp (\mathrm{i} 2 \Delta \beta z)+\delta n_{z}^{*}\right] E_{-0}+\delta n_{x+y}^{*} E_{-\mathrm{h}}\right\}, \\
\frac{\mathrm{d} E_{-0}}{\mathrm{~d} z} & =\mathrm{i} \frac{k_{\mathrm{m}}}{4 \cos \theta_{\mathrm{B}}}\left\{\delta n_{x-z}^{*} E_{0}+\left[M_{z} \exp (-\mathrm{i} 2 \Delta \beta z)+\delta n_{z}\right] E_{\mathrm{h}}+2 \delta n_{0} E_{-0}+\delta n_{x}^{*} E_{-\mathrm{h}}\right\}, \\
\frac{\mathrm{d} E_{-\mathrm{h}}}{\mathrm{d} z} & =\mathrm{i} \frac{k_{\mathrm{m}}}{4 \cos \theta_{\mathrm{B}}}\left\{\left[M_{z} \exp (-\mathrm{i} 2 \Delta \beta z)+\delta n_{z}\right] E_{0}+\delta n_{x+z} E_{\mathrm{h}}+\delta n_{x} E_{-0}+2 \delta n_{0} E_{-\mathrm{h}}\right\} .
\end{aligned}
$$

In the Eqs. (6a)-(6d), the parameters are defined as follows $\delta n_{0}=n_{\alpha}\left(\left|E_{0}\right|^{2}+\left|E_{\mathrm{h}}\right|^{2}+\left|E_{-0}\right|^{2}+\right.$ $\left|E_{-\mathrm{h}}\right|^{2}, \delta n_{z}=2 n_{\alpha}\left(E_{\mathrm{h}}^{*} E_{-0}+E_{0}^{*} E_{-\mathrm{h}}\right), \delta n_{x}=2 n_{\alpha}\left(E_{0} E_{\mathrm{h}}^{*}+E_{-0}^{*} E_{-\mathrm{h}}\right), \delta n_{x+z}=2 n_{\alpha}\left(E_{\mathrm{h}}^{*} E_{-\mathrm{h}}\right)$, $\delta n_{x-z}=2 n_{\alpha}\left(E_{0} E_{-0}^{*}\right), M_{z}=2 n_{z} / n_{0}$, and $2 \Delta \beta=2 k_{\mathrm{m}}\left(\cos \theta-\cos \theta_{\mathrm{B}}\right)=-2 \eta k_{\mathrm{m}} \sin \theta_{\mathrm{B}}$. Where 
$\theta$ is the incident angle of incoming radiation, and $\eta$ represents the angular deviation from the Bragg angle $\theta_{\mathrm{B}}$.

The four diffracted waves at their exit boundaries are determined by the field equations, the material equations, and the boundary conditions, $E_{0}(0)=E_{\text {in } 1}, E_{\mathrm{h}}(0)=E_{\text {in } 2}, E_{-0}(-l)=0$, $E_{-\mathrm{h}}(-l)=0$. For convenience, we define a parameter $\gamma$ as $\left|E_{i n 1}\right|^{2} /\left|E_{\text {in2 } 2}\right|^{2}$, and make $0 \leq \gamma \leq 1$. Note in the case of $\gamma=0$, the optical system is an ordinary DFB system, and the resonance is mainly obtained through the DFB. Otherwise, when $\gamma$ is of order of 1 , an ID 2D superlattice will be constructed, and the resonance is obtained through IM feedback and DFB. In an ID 2D nonlinear superlattice, a suitable axis along $x$-direction can be selected to make the two incident waves in the same propagating phase. That is to say, four diffracted waves intensities at their exit boundaries are independent on the transmission phases of the two incident waves. So, one can neglect the influence of the phases of the two incident waves, and always make boundary conditions satisfy $E_{0}(0)=\sqrt{I_{\text {in }} /(1+\gamma)}, E_{\mathrm{h}}(0)=\sqrt{\gamma I_{\text {in }} /(1+\gamma)}, E_{-0}(-l)=0, E_{-\mathrm{h}}(-l)=0$. Here $I_{\text {in }}=\left|E_{\text {in } 1}\right|^{2}+\left|E_{\text {in2 }}\right|^{2}$ is total incident intensity.

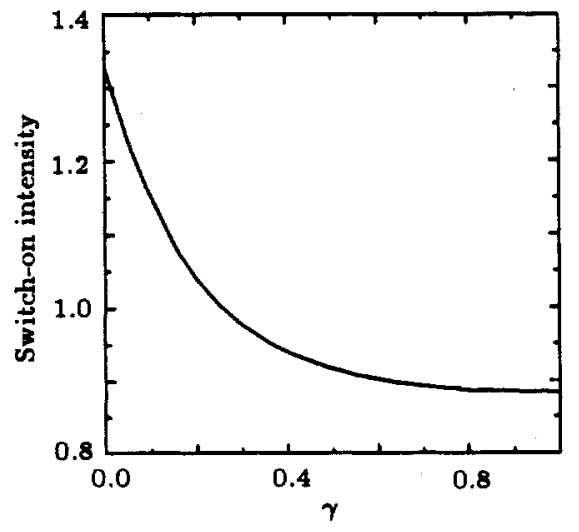

Fig. 3. The $\gamma$-switch-on relation obtained by numerical calculations. The structure parameters are the same as in Fig. 2.

Because of the complexity of these equations, the solutions must be achieved by numerical method. The input-output relation is plotted in Fig. 2 for an ID 2D nonlinear superlattice with $\triangle \beta=0$, $M_{z}=2 \times 10^{-4}, k_{\mathrm{m}} l / \cos \theta_{\mathrm{B}}=3.5 \times 10^{4}$, and $\gamma=0.3$. At lower incident intensity, the transmitted intensities $I_{0}$ and $I_{\mathrm{h}}$ are weak. Once above the switch-on intensity (critical intensity), the transmitted intensities jump from the lower intensities to higher intensities in a discontinuous way. Thus in the case of two incident waves, the output intensities can be bistable. It should be mentioned that the bistability discussed in Ref. 8 is related to the transmission transition in the allowed band. As shown in Fig. 2, the bistability discussed here is related to the transition between the allowed transmission and the forbidden

transmission. At a certain total incident intensity, the larger the $\gamma$, the stronger the IM feedback is. Then the switch-on intensity becomes lower. We plot $\gamma$-switch-on relation in Fig. 3 . It is seen from this plot that the switch-on intensity will decrease with $\gamma$. Therefore, in the general case, a relatively low bistable threshold exists in the ID 2D nonlinear superlattice due to contribution of the IM mechanism.

In conclusion, we have demonstrated that an ID 2D nonlinear superlattice constructed originally from a $1 \mathrm{D}$ superlattice by two incident waves is bistable. The optical bistability originates from the IM and DFB mechanism. The threshold for the bistability is relatively low due to the IM feedback.

\section{REFERENCES}

1] H. G. Winful, J. H. Marburger and E. Garmire, Appl. Phys. Lett. 35 (1979) 379.

[2] W. Chen and D. L. Mills, Phys. Rev. Lett. 58 (1987) 160.

3 W. Chen and D. L. Mills, Phys. Rev. B 36 (1987) 6269.

[4] C. M. de Sterke and J. E. Sipe, Phys. Rev. A 42 (1990) 2858.

5 V.M. Agranovich, S. A. Kiselev and D. L. Mills, Phys. Rev. B 44 (1991) 10917.

[6] M. Scalora, J. Dowling, C. M. Bowden and M. J. Bloemer, Phys. Rev. Lett. 73 (1994) 1368.

77. M. D. Tocci, M. J. Bloemer, M. Scalora, J. Dowling and C. M. Bowden, Appl. Phys. Lett. 66 (1995) 2324.

8 B. Xu and N. B. Ming, Phys. Rev. Lett. 71 (1993) 1003.

[9] B. Xu and N. B. Ming, Phys. Rev. Lett. 71 (1993) 3959. 